\title{
Negotiation Transactions: An Approach to Increase the Automation of Workflows
}

\author{
Juha Puustjärvi \\ Department of Computer Science \\ P. O. Box 26 (Teollisuuskatu 23) \\ FIN-00014 UNIVERSITY OF HELSINKI \\ Finland \\ e-mail: puustjar@cs.helsinki.fi
}

\begin{abstract}
This paper investigates how the automation level of transactional workflow can be increased. The proposed model enables to automate the business processes where dealing is made by first requesting the offers and then making the deal based on the offers. In order to automate the negotiation of many concurrent participants located at different autonomous sites, the negotiation transaction model is introduced. The main idea behind the negotiation transaction model is to carry out the negotiation by a protocol, called the 2PN-protocol, which has the same kind of execution structure as the 2PC-protocol has, but those differ in that the messages of the $2 \mathrm{PN}$-protocol have workflow specific semantics. Further, in order to avoid the problems caused by the long duration of negotiation transactions, we have designed a new local transaction model, called the 2P-transactions, which are also useful independently of the negotiation transactions.
\end{abstract}

\section{Introduction}

Research in workflow models has been originally motivated by the need to automate office paper processing. Later on, extended transaction models $[9,10,26]$ as well as transactional workflow models (e.g., $[28,3,12,27,18,30]$ ) have been introduced in order to achieve the correctness and reliability of workflows. Also cooperative transaction models (e.g., $[22,17,20,15,19]$ ) have been developed for supporting cooperative work in centralized environment. These models allows users to cooperate only by successive tasks, but negotiation-like cooperation cannot be modelled. On the other hand, with groupware systems [8] users (humans) can cooperate spontaneously without predefined protocols. Such cooperation is necessary for many groupware applications, and integrating such features also for workflow systems would be highly appropriated.

Further, in many distributed applications the users of autonomous local systems have to cooperate. Typically cooperation is based on a predefined practice, but such cooperation cannot be automated as there are neither model nor methods to carry out the cooperation. For example, the business processes where dealing is made by first requesting the offers and then making the deal are very common 
in practice. Our goal then is to develop appropriate concepts and methods to automate such cooperative processes.

The corner stone for our analysis is a protocol, called the 2-phase negotiation protocol, or the $2 P N$-protocol for short. The $2 \mathrm{PN}$-protocol is similar to the $2 \mathrm{PC}$ protocol [2] in that it consists of the request phase and decision phase, but those differ in that the spectrum of the negotiation with the $2 \mathrm{PN}$-protocol is not restricted to atomic commitment.

Essentially, the 2PN-protocol connects the components of a distributed workflow through negotiation transactions. Further, in order to avoid the problems related to long lasting transactions, we have designed a local transaction model, called 2-phase transaction, or $2 P$-transaction for short, which is a subtransaction of a negotiation transaction. A 2P-transaction is executed in two phases, each consisting of a local transaction. Intuitively, the function of the first phase is to ensure that the second phase can be executed, though the exact function of the second phase is not known until it begins.

The remainder of the paper is organized as follows. Section 2 presents our view of workflow modularity, the notion of a transactional workflow, and the example system which is used through the paper to motivate and illustrate our proposed notions. Section 3 presents the structures of a negotiation workflow and a negotiation transaction, and particularly how those are linked by the $2 \mathrm{PN}$ protocol. Section 4 discusses the implementation of the 2P-transactions. Section 5 concludes the paper and proposes some new applications of the $2 \mathrm{P}$-transactions.

\section{Modelling Workflows}

Our view of transactional workflows is twofold: we focus on modular workflow structure $[24,25]$ as well as the structure of negotiation transactions. Further, the intertwinement of these two structures is our main topic. Ideally,

- the semantics of negotiation is included in workflow modules (tasks),

- while the negotiation is carried out by the 2PN-protocol, and

- the updates resulted by the negotiation is executed by the negotiation transaction.

So, the modifications on the negotiation practice is transparent to the negotiation transactions. This is very important aspect as workflow modifications may occur frequently, and for this reason, workflow system should provide fast redesign and reimplementation properties.

In order to prepare for the later sections, we now shortly consider the concepts of modular workflows and transactional workflow. In addition, we will present an example system, which will be used throughout the paper to illustrate our proposed notions.

\subsection{Modularity}

Fundamentally, a workflow is a collection of tasks organized to accomplish some business process. Again each task defines some work to be done. For example, a 
form processing, procedure, email or a transaction may be a task. A workflow ties a set of task together by specifying the execution dependencies and the data flow between the tasks. For example, a task may not begin before a particular previously started task commits. Such constraints are usually specified by triggers $[7,6]$, Petri nets [16] or by finite state automaton [1]. In contrast with our prosed negotiation workflows, execution dependencies and data flow is carried out by the $2 \mathrm{PN}$-protocol. On the other hand, as a negotiation workflow may be a subworkflow of an other workflow, our approach does not exclude other methods in enforcing tasks' execution dependencies.

From architectural point of view we model workflows by a modular structure consisting of a workflow pool and task pool (Fig. 1.)

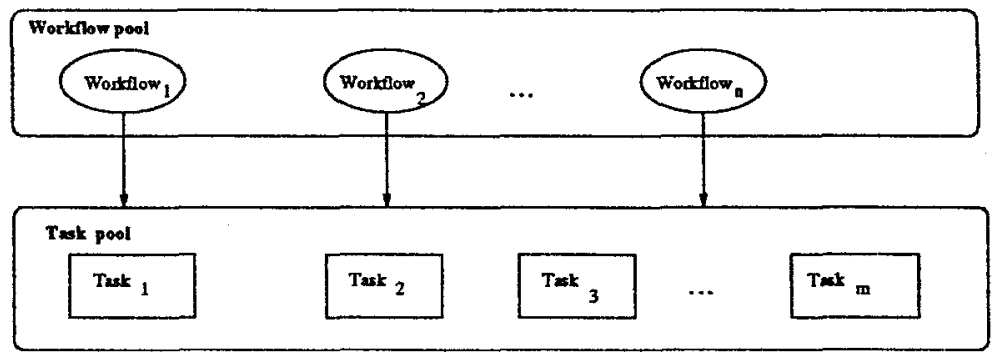

Fig. 1. Modular component architecture.

Each task belongs at least to one workflow. If a tasks belongs into several workflows, then the task is reused. Basically, task reusability avoids redundant design of workflows. This is very important aspect as large workflow applications are typically define by combining existing applications.

We still extend the notion of task reusability by allowing workflows as well to be reusable. Particularly, our proposed negotiation workflows may be independent workflows or as a component (subworkflow) of an other workflow.

Equally modular workflow specification applies to distributed environment. Now each site has the workflow pool and the task pool, but a task may also belong to the workflow locating in the workflow pool of an other site. Especially, this is the case with each negotiation workflow as all it's tasks are located at different sites.

\subsection{Transactional Workflows}

Correctness and reliability of workflows can be achieved by transactional workflows. Correctness means that the concurrent execution of concurrent transactions and workflows are interleaved in a way that incorrect results such as lost update or inconsistent retrieval cannot occur. Reliability means that both tasks and workflows are recoverable in the event of failures.

Correctness and reliability of a workflow can be achieved by specifying it as an extended transaction. This means that 
- the tasks of the workflow correspond the subtransactions of the extended transaction,

- the workflow obeys the same execution dependencies as the corresponding extended transaction, and

- the workflow has the same transactional properties as the corresponding extended transaction model (e.g., atomicity based on compensating transactions).

Due to the limited applicability of extended transaction models, no single extended transaction model can be used to model all workflows. So, as it is proposed in [13], workflow system should be able to support various extended transaction models.

Furthermore, we argue that the proposed extended transaction models fit to the requirements of transactional workflows as far as there are no negotiation between the subtransactions of an extended transaction. The reson is that execution dependencies are not suitable for specifying negotiation. This gave us an impulse to develop negotiation transactions, but before their introduction we will motivate their need by the following well known example of trip planning $[14,9,4]$.

\subsection{The Trip Planning Example}

The trip planning example [14] was originally presented to show that the subtransactions of a transaction may have different kind of transactional requirements, e.g., some subtransactions may be undone or redone while other subtransactions may be deferred but not undone. Later on, alternative subtransactions [9] and contingency transactions [4] has been motivated by the example. Again, we motivate the need of negotiation transactions by the example.

In the trip planning example a transaction may consist of the following subtransactions or actions:

A1 Customer calls the travel agent to reserve a trip.

A2 Agent negotiates with airlines to order a ticket for a flight.

A3 Agent negotiates with hotels to reserve a room.

A4 Agent negotiates with car rental companies for car reservation.

A5 Agent receives tickets and reservations and then gives them to the customer.

For simplicity we assume that there are only two airlines, say Finnair and SAS, and two hotels, say Sheraton and Hilton, to be consulted. The travel agent can then order a ticket from either Finnair or SAS, and respectively the agent can reserve a room either from Sheraton or Hilton. Hence, using alternative subtransactions one can specify that if the subtransaction reserving a ticket from Finnair fails, then the subtransaction which tries to make the reservation from SAS is executed, or vice versa.

We still increase the automation and expression power of the trip planning system by negotiation workflows and negotiation transactions. This can be simply 
motivated by assuming that a customer may not prefer any of the airlines or hotels, but rather wants to make the decision (reservation) based on a choice criterion, (e.g., choose the cheapest one). Hence, the choice criterion is evaluated during the execution of the negotiation transaction. And most important, the evaluation is done by the negotiation workflow - not the negotiation transaction. For this reason, as the criterion or its evaluation principles changes, the modifications have to be done only on the negotiation workflow - not on the negotiation transaction.

\section{The Model}

This section presents the structures of the negotiation workflow and the negotiation transactions, and particularly, how those are linked together by the $2 \mathrm{PN}$ protocol. Our perspective on negotiation transactions is rather focused on their functionality than on their formal specification, which could be given for example by the ACTA-framework [5] or by deontic logic [29].

\subsection{Negotiation Workflow and Negotiation Transaction}

A negotiation workflow is comprised of one coordination task and one or more participant tasks (Fig. 2.). These tasks are located at the task pools of different sites.

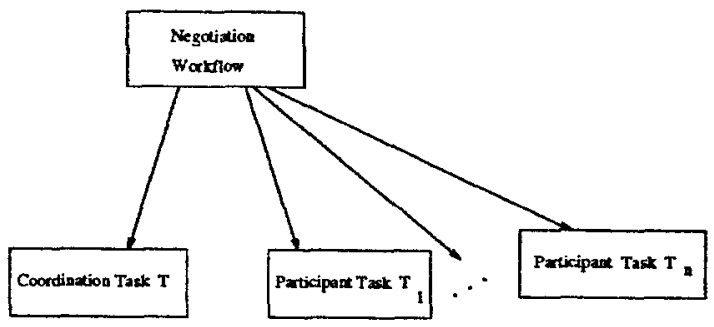

Fig. 2. The tasks of a negotiation workflow.

Ideally, the coordination task negotiates with the participant tasks to make a deal. So, the actions A2, A3 and A4 of the trip planning example can be modelled as negotiation workflows (Fig. 3). The home site of these negotiation workflows is the site of their coordination task, i.e., the home site of each of these negotiation workflow is the site of the travel agent.

A negotiation workflow may be an independent workflow or a subworkflow of an other workflow. For example, A2, A3 and A4 are subworkflows, and are therefore denoted by double outlines in the figure. In addition, as the workflow trip planning may be independent workflow or a subworkflow of an other workflow, it is also denoted by double outline. The arcs denote the execution constraints of the components. 


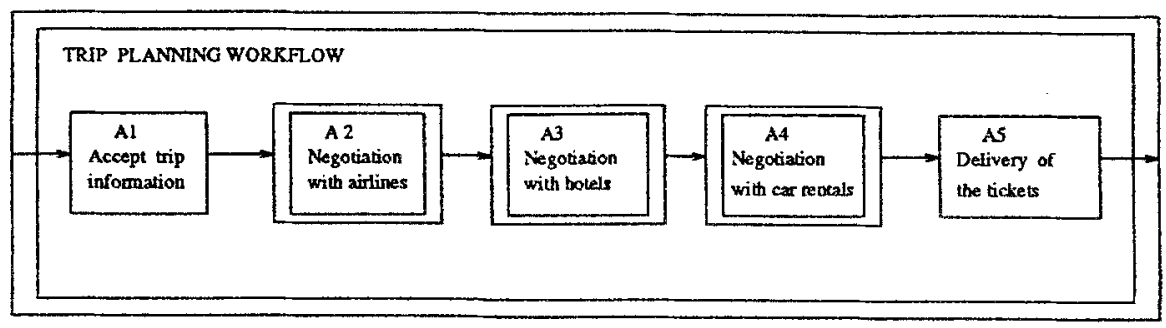

Fig. 3. The modular structure of the workflow trip planning.

A negotiation transaction is comprised of one coordination transaction and one or more 2P-transactions (Fig-4.)

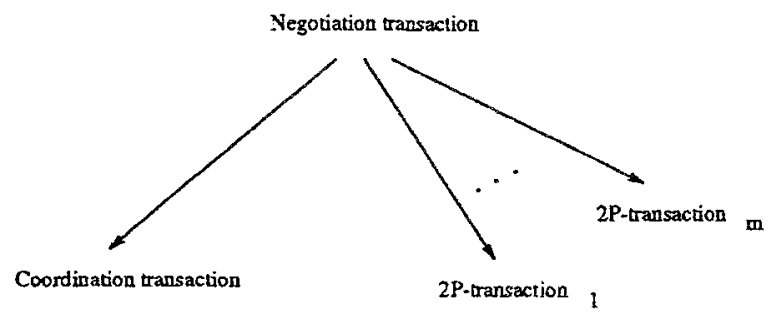

Fig. 4. The components of a negotiation transaction.

Intuitively, the coordination transaction is an agent of the coordination task in the sense that it performs in a reliable and correct way the database operations of the coordination task (Fig. 5). Respectively, each $2 P$-transaction is an agent of a participant task. These transactions are synchronized by the messages of the $2 \mathrm{PN}$-protocol. Such communication is denoted by the broken lines in Fig. 5 .

\section{$3.2 \quad 2 P$-transactions}

A 2P-transaction is started in the beginning of the negotiation, and it will be active until the end of the negotiation. Due to the relative long communication delays between the sites, the negotiation will take rather long a time, giving rise that $2 \mathrm{P}$ transactions will be long lasting. Similarly, in heterogeneous distributed database systems [23] the implementation of atomic distributed transactions requires long lasting local subtransactions, which in turn violate local system autonomy and decrease local system performance. For this reason, in many distributed systems (multidatabases) atomic distributed transactions are not supported, but rather some form of compensation is used to ensure semantic atomicity of distributed transactions [11].

To avoid the problems of long lasting local transactions, we have developed a specific structure and semantic for 2P-transactions. Particularly, each 2Ptransaction decomposes into one preliminary transaction and to two or more 


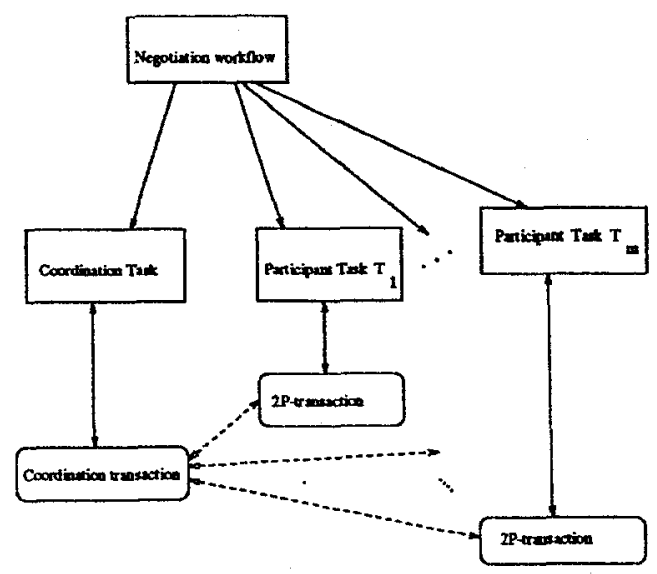

Fig. 5. The involvement of the negotiation workflow and the negotiation transaction.

complementary transactions (Fig. 6). With each 2P-transactions at least two complementary transactions are attached, but when a $2 \mathrm{P}$-transaction is executed only one of the complementary transactions is executed. Consequently a $2 \mathrm{P}$ transaction consists of the two phases: in the first phase the preliminary transaction is executed and in the second phase a complementary transaction is executed, hence the name 2-phase transaction.

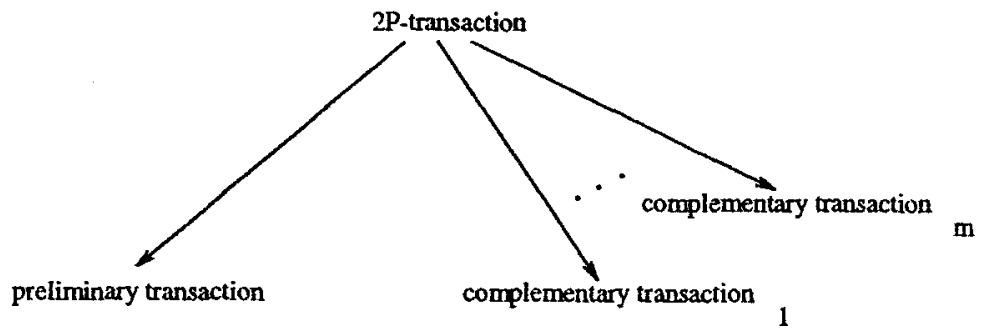

Fig. 6. The components of a $2 \mathrm{P}$-transaction.

The preliminary transaction is executed at the beginning of the negotiation, and based on the decision of the negotiation the corresponding complementary transaction is executed. So during the negotiation, which may take rather long a time due to the communication delays, no local transaction has to hold resources, e.g., locks on data items.

\subsection{The 2PN-protocol}

The 2PN-protocol has two functions. First, it coordinates the negotiation, and second, it ensures reliability in the presence of failures. For the clarity we first consider the protocol assuming that there are no failures and then (in the next 
section) we present the exact protocol, which is resilient for system and communication failures.

To carry out the negotiation there is a process at the site of the coordination task and the sites of the participant tasks. The process at the site of the coordination task is called the coordinator, and the processes at the sites of the participant tasks are called the participants. The coordinator knows the names of the participants, and so it can send them messages. The participants know the name of the coordinator, but they don't know each other.

Assuming that there are no failures the 2PN-protocol goes as follows:

1. The coordination task activates the coordinator, and thus starts the 2PNprotocol, by passing the REQUEST-messages to it. Then the coordinator sends a REQUEST-message to all participant. The messages may or may not be equal.

2. When a participant receives a REQUEST-message, it first activates the participant task by passing the message to it, and after processing the message it passes the RESPONSE-message to the participant. If the participant task accepted the request, then the participant starts the preliminary transaction, and if it commits then the participant sends the RESPONSE-message to the coordinator. Else it passes the information of the abort to the participant task.

3. The coordinator collects the RESPONSE-messages and passes the messages to the coordination task. After their processing, the coordination task passes the DECISION-messages to the coordinator, which sends these participant specific DECISION-messages to the participants. (For illustrative purposes we say that the DECISION-message is negative, if the coordinator did not accepted the response, and otherwise it is positive.)

4. Each participant that accepted the request waits a DECISION-message from the coordinator. After receiving the DECISION-message the participant passes it to the participant task, which after processing it outputs the identifier of the complementary transaction to be executed, and then the participant starts the complementary transaction.

In Fig. 7 we have illustrated by a state diagram the synchronization of the protocol messages and the execution of the preliminary and the complementary transactions. The diagram represents the states of the participant in the case of two complementary transactions (a positive and a negative complementary transaction). With respect to the negotiation of a ticket for a flight we can give the following interpretation for the diagram.

First, the coordinator sends flight-id/seats-request, which identifies the flight and the number of requested seats. Then the participants executes the preliminary reservation (preliminary transaction) and sends the flight-id-price/seats-response (positive response) or flight-id/not-available-response (negative response) to the coordinator. Finally the coordinator sends participant specific flight-id/passengers message(s) (positive DECISION-message) to the participant(s) offering the flights to the lowest price, and to the other participants it sends flight-id/nodeal-messages (negative DECISION-message). 


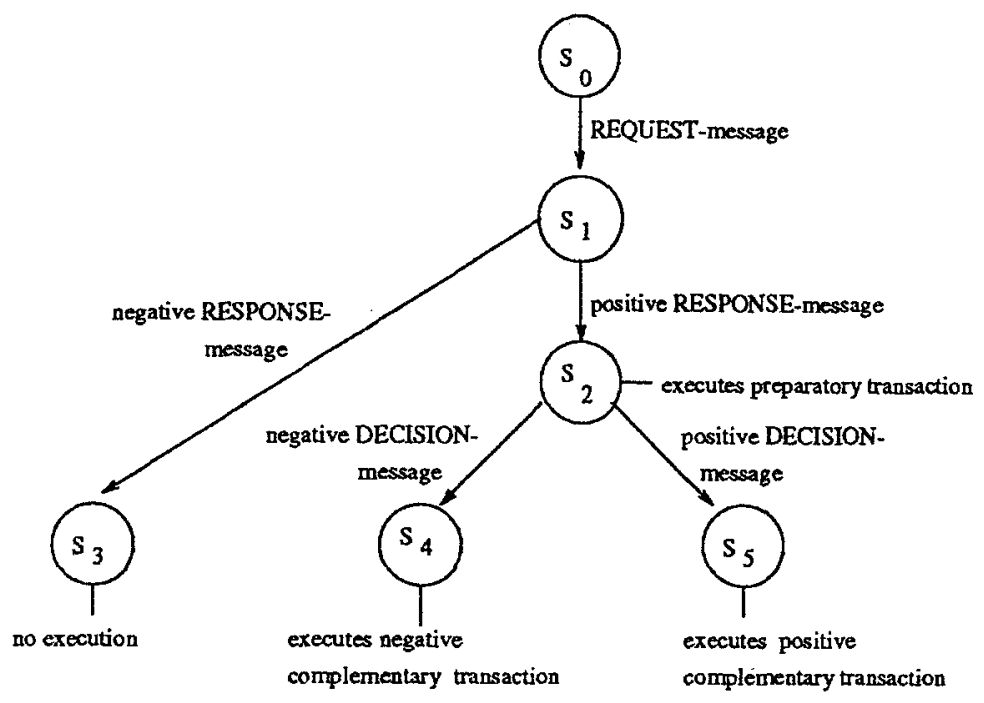

Fig. 7. The states of the participant.

\subsection{Reliability}

As in practice the system and communication failures may occur, also the 2PNprotocols should tolerate them. Accordingly we will require that, if all existing failures are repaired and no new failures occur for sufficiently long, then the $2 \mathrm{PN}$-protocol will eventually be executed.

There are two reason why the protocol described in the previous section does not tolerate communication and system failures:

- First, at various points of the protocol either the coordinator or the participants must wait for messages before proceeeding, but messages may not arrive due to a failure giving rise that those may be waiting forever. For this reason we have to add timeout actions to the protocol.

- Second, when the coordinator or a participant recovers from a failure it has to reach the decision. This requires that the coordinator and the participants have to keep some information in the log (stable storage).

The following $2 \mathrm{PN}$-protocol tolerates both communication and system failures.

\section{Coordinator:}

send REQUEST-message to all participants;

write start-QPN record in $\log$;

wait for RESPONSE-messages from the participants;

if the messages have received from all participants or timeout expired then Begin

pass the RESPONSE-messages to the coordination task; 
according to the output of the coordination task;

write decision-records in log;

execute the COORDINATION TRANSACTION;

end;

send DECISION-messages to participants;

else write DECISION-message "no RESPONSE-message received" in log; return

\section{Participant:}

wait for REQUEST-message from the coordinator;

on receiving REQUEST-message activate the participant task by

passing the REQUEST-message to it;

according to the output of the participant task

write response-record in log;

if the RESPONSE-message is positive then

begin

execute the PRELIMINARY TRANSACTION;

if the PRELIMINARY TRANSACTION committed then begin

send RESPONSE-message (positive message) to the coordinator;

wait for DECISION-message from the coordinator;

on timeout initiate termination protocol;

write decision-record in log;

pass the DECISION-messages to the participant task;

according to the response of the participant task

execute a COMPLEMENTARY TRANSACTION;

end;

else activate the participant task by passing the Abort-message to it;

end;

else send RESPONSE-message (negative message) to the coordinator;

return

The period between the participant sent a positive RESPONSE-message and the participant received the DECISION-message is the uncertainty period for that participant. During the uncertainty period the participant does not know whether it's positive RESPONSE-message is accepted. If the participants in it's uncertainty period is interrupted by a timeout, then the participant must consult with the coordinator to find out the decision. This consultation is carried out by the following termination protocol of the $2 \mathrm{PN}$-protocol.

\section{Initiator's algorithm:}

start: send DECISION-request to the coordinator;

wait for DECISION-message;

on timeout goto start;

on receiving DECISION-message return; 
Coordinator's algorithm:

wait for DECISION-request from any participant;

send DECISION-message to the participant;

return

As we have assumed that the coordination transactions, preliminary transactions and complementary transactions are executed as local transactions in the local systems providing the ACID-properties [2], we do not have to take care of their reliability.

\section{Implementing 2P-transactions}

Before considering the methods to implement $2 \mathrm{P}$-transactions we will specify the correctness of $2 \mathrm{P}$-transactions. We say that a $2 \mathrm{P}$-transaction is correct, if it satisfies the following constraints.

- The commitment of it's preliminary transaction implies that any of it's complementary transaction cannot be aborted as a result of the violation of an integrity constraint.

- The database is in a consistent state after the execution of the preliminary transaction, i.e., the uncertainty period is made visible to other transactions.

A general approach for enforcing these constraints is to model the uncertainty period, by executing a separate transaction, which make the uncertainty period visible to other transactions. For example, with respect to the reservations in the trip planning example, we should make the difference between a preliminary reservation and a real reservation. Hence, the preliminary transaction would be the execution of the preliminary reservation, and if the positive RESPONSE is received then the preliminary reservation is changed into real reservation, and if the negative RESPONSE is received then the preliminary reservation is canceled.

On the other hand, if the integrity constraint(s) which may abort the execution of the complementary transaction is based on a comparison operator, then we can enforce $2 \mathrm{P}$-transactions by introducing the notion of a shadow constraint. The general form of a shadow constraint is the following:

<data-object $><$ comparison-operator $>\langle$ numerical-value $>$

Intuitively, a shadow constraint is a dynamic integrity constraint such that only the 2P-transactions are allowed to modify it, and thus control the execution of other transactions. The initial state of a shadow constraint equals to the corresponding integrity constraint, and so the integrity constraint having a shadow constraint must also be of the form above. 
Precisely, the preliminary and the complementary transactions execute increment and decrement operations on the numerical-value of the shadow constraint according to the following rules:

- If the preliminary transaction decreases the numerical-value by $i$, then the complementary transaction of the same $2 \mathrm{P}$-transaction increases the numerical value by $i$.

- If the preliminary transaction increases the numerical-value by $i$, then the complementary transaction of the same $2 \mathrm{P}$-transaction decreases the numerica value by $i$.

That is, the complementary transaction compensates the effect of the preliminary transaction. And, most important, in the complementary transaction the update of the numerical value and the update of the database are executed in this order, and as an atomic unit. To illustrate this assume that due to the aircraft type there is an integrity constraint stating that the reserved seats for a flight is allowed to be at most 100 . Thus, at each time a new reservation is made the object reservedseats is increased accordingly and the reservation is accepted as far as the value of the object reserved-seats does not exceed 100.

Now, assume that an airline receives the REQUEST-message concerning five reservations. So, if the value of reserved-seats does not exceed 95 , the preliminary transaction is executed and the positive response message is sent. In this case the preliminary transaction has decreased the shadow constraint, say $S C$, by 5 . If there are no other active 2P-transaction concerning to the seats of the same flight, then the shadow constraint will be "SC $\leq 95$ ". On the other hand, if there is such an other 2P-transaction, say negotiating of 2 reservations, then the shadow constraint will be " $S C \leq 93$ ". This means that other transactions are allowed to do reservations only as far as there are less than 93 reservations, but otherwise only the complementary transactions of the two 2P-transactions are allowed to modify the shadow constraint, and thus only those transactions can make new reservations. Further, as the complementary transaction is atomic, no other transaction can make the reservation as a result of the increment of the shadow constraint.

Logically, our proposed shadow constraints have similarities with escrow locking [21], which is designed to deal with arithmetic operations on hotspot data. The idea behind escrow locking is to preserve the truth of a predicate on a data item between the time the transaction first reads the data item and the time the predicate is evaluated at committing the transaction. Our approach differs from this approach with two aspects. First, instead of predicates we evaluate only integrity constraints. Second, with $2 \mathrm{P}$-transactions the truth of the integrity constraint (predicate) is enforced the time between two separate transaction not a time interval within the execution of one transaction.

\section{Conclusions}

The aim of our proposed negotiation workflow and negotiation transactions is to automate negotiation-like tasks which so far has required human intervention. 
Particularly, those enables to automate the business processes where dealing is made by first requesting the offers from the producers. On the other hand, by the trip planning example we have shown that by replacing alternative subtransactions by negotiation transactions, we can increase the expression power of the model. Further, it obvious that $2 \mathrm{P}$-transactions are also useful when used independently, i.e., apart from the $2 \mathrm{PN}$-protocol. To see this assume that an enterprise makes an offer of products for a customer, and after a time the customer either accepts or refuses the offer. Ensuring that the offered products are still safe when the customer responses, these operations should be executed as one transaction, giving rise that the data items are locked for a long time, i.e., we encounter the same problem which impulsed us to introduce the notion of $2 \mathrm{P}$-transdactions.

\section{References}

1. M. Attie, M. Singh, A. Sheth, and M. Rusinkiewicz. Specifying and enforcing intertask dependencies. In The 19th International Conference on VLDB, 1993.

2. P. Bernstein, V. Hadzilacos, and N. Goodman. Concurrency Control and Recovery in Database Systems. Addison-Wesley, 1987.

3. Y. Breitbart, A. Deacon, H.-J. Schek, A. Sheth, and G. Weikum. Merging application-centric and data-centric approaches to support transaction-oriented multi-system workflows. Sigmod Record, 22(3), September 1993.

4. A. Buchmann, M. Özsu, M. Hornick, D. Georgakopoulos, and F. Manola. A transaction model for active distributed object system. In A.K. Elmagarmid, editor, Database Transaction Models for Advanced Applications, chapter 5. Morgan Kaufmann Publishers, 1992.

5. P. Chrysanthis and K. Ramamrithan. Acta: The saga continues. In A.K. Elmagarmid, editor, Database Transaction Models for Advanced Applications, chapter 10. Morgan Kaufmann Publishers, 1992.

6. U. Dayal, M. Hsu, and R. Ladin. A transaction model for long-running activities. In The 17th International Conference on VLDB, 1991.

7. U. Dayl, M. Hsu, and R. Ladin. Organizing long-running activities with triggers and transactions. In ACM SIGMOD International Conference on Management of Data, 1990.

8. C. Ellis, S. Gibbis, and G. Rein. Groupware: Some issues and experiences. Communications of the ACM, 34(1), January 1991.

9. A. Elmagarmid, Y. Leu, W. Litwin, and M. Rusinkiewicz. A multibase transaction model for interbase. In The 16th International International Conference on VLDB, 1990.

10. H. Garcia-Molina, D. Gawlick, J. Klein, K. Kleissner, and K. Salem. Modeling long-running activities as nested sagas. IEEE Data Engineering Bulletin, 14(1), March 1991.

11. H. Garcia-Molina and K. Salem. Sagas. In ACM SIGMOD International Conference on Management of Data, 1987.

12. D. Georgakopoulos and M. Hornick. A framework for enforceable specification of extended transaction models and transactional workflows. Journal of Intelligent and Cooperative Information Systems, September 1994. 
13. D. Georgakopoulos, M. Hornick, F. Manola, M. Brodie, S. Heiler, F. Nayeri, and B. Hurwitz. An extended transaction environment for workflows in distributed object computing. IEEE Data Engineering Bulletin, 16(2), June 1993.

14. J. Gray. The transaction concept: Virtues and limitations. In The 7 th International Conference on $V L D B, 1981$.

15. W. Harrison, H. Ossher, and P. Sweeney. Coordinating concurrent development. In Proceedings of the Conference on Computer-Supported Cooperative Work, 1990.

16. K. Jensen. Colored Petri Nets. Springer-Verlag, 1991.

17. G. Kaiser and C. Pu. Dynamic restructuring of transactions. In A.K. Elmagarmid, editor, Database Transaction Models for Advanced Applications, chapter 8. Morgan Kaufmann Publishers, 1992.

18. J. Miller, A. Sheth, K. Kochut, and X. Wang. Corba-based run-time architectures for workflow management systems. Journal of Database Management, 7(1), Winter 1996.

19. K. Narayanaswamy and K. Goldman. "Lazy" consistency: A basis for cooperative software development. In Proceedings of the Conference on Computer-Supported Cooperative Work, 1992.

20. M. Nodine, S. Ramaswamy, and S. Zdonik. A cooperative transaction model for design databases. In A.K. Elmagarmid, editor, Database Transaction Models for Advanced Applications, chapter 3. Morgan Kaufmann Publishers, 1992.

21. P.E. O'neil. The escrow transactional method. ACM Transactions on Database Systems, 11(4), December 1986.

22. C. Pu and N. Hutchinson. Split transactions for open ended activities. In The 14th International Conference on VLDB, 1988.

23. J. Puustjärvi. Distributed management of transactions in heterogeneous distributed database systems. BIT, 31(3), 1991.

24. J. Puustjärvi, H. Tirri, and J. Veijalainen. Concurrency control for overlapping and cooperative workflows. Bulletin of the Technical Committee on Operating Systems and Application environments (TCOS), 8(1), Spring 1996.

25. J. Puustjärvi, H. Tirri, and J. Veijalainen. Managing overlapping transactional workflows. In The Sth International Conference on Advanced Information Systems Engineering (CAiSE'96), 1996.

26. M. Rusinkiewicz, A. Elmagarmid, Y. Leu, and W. Litwin. Extending the transaction model to capture more meaning. SIGMOD Record, 19(1), 1990.

27. M. Rusinkiewicz and A. Sheth. Specification and execution of transactional workflows. In W. Kim, editor, The Object Model, Interoperability, and Beyond. Addison-Wesley, 1994.

28. H. Wähter and A. Reuter. The contract model. In A.K. Elmagarmid, editor, Database Transaction Models for Advanced Applications, chapter 7. Morgan Kaufmann Publishers, 1992.

29. H. Weigand and $\mathrm{E}$. Verharen. Interoperable transactions in businessss models-a structured approach. In The 8th International Conference on Advanced Information Systems Engineering (CAiSE'96), 1996.

30. D. Worah and A. Sheth. What do advanced transaction models have to offer for workflows? In Proceedings of the International Workshop on Advanced Transaction Wodels and Architectures (ATMA), 1996. 